\title{
Design algorithm for generatrix profile of cylindrical crowned rollers
}

\author{
Spiridon Creţu* \\ Technical University" Gheorghe Asachi" of Iaşi, Department of Mechanical Engineering, 63, D. \\ Mangeron St., Iaşi, Romania
}

\begin{abstract}
The cross-section of roller profile controls the pressure distribution in the contact area and radically affects the roller bearings basic dynamic load rating and rating lives. Today the most used roller profiles are the logarithmic profile and cylindrical-crowned (ZB) profile. The logarithmic profile has a continuous evolution with no discontinuities till the intersection with the end fillet while ZB profile has two more discontinuities at the intersections points between the crowning circle and straight line generatrix. Using a semianalytical method, a numerical study has been carried out to find the optimum ZB profile for rollers incorporated in cylindrical rollers bearings. The basic reference rating life $\left(L_{10_{-}}\right)$has been used as optimization criterion.
\end{abstract}

\section{Introduction}

Design changes which reduce material stresses appeared to be the main possibilities to improve roller bearing lives within the same envelope dimensions. The cross -section of roller profile controls the pressures distribution in the contact area and radically affects the bearing basic dynamic load rating and rating lives, [1-3]. Consequently, the primary target was to reduce stresses in the roller raceway contact by modifying roller profile.

The diversity of crowning profiles includes: the single straight line with end fillets, single arc or a combination of multiple arcs, cylindrical-crowned (ZB), Figure 1, and logarithmic. Reviewing papers [4-7] have pointed out that the contact pressures tends to be significantly higher around the profile's discontinuities than the pressures attained in the middle of the contact area. The edge effect is considerably amplified when the bearing operates with a misalignment angle $\psi$ between axes of the outer and inner rings [2-4].

\section{End relief set up}

The size of cylindrical roller is described by diameter $D_{w}$ and length $L_{w}$. The cylindrical-crowned profile is defined by two parameters: the value $\mathrm{ZK}$ of the end relief, and value $\mathrm{b}$ representing the length of circular profile, Figure 1. For a particular size $\left(D_{w} * L_{w}\right)$ of cylindrical roller, the end relief $\mathrm{Z}_{\mathrm{K}}$ of $\mathrm{ZB}$ profile is established considering the values provided by the chosen logarithmic profile.

\footnotetext{
*Corresponding author: spcretu@tuiasi.ro
} 


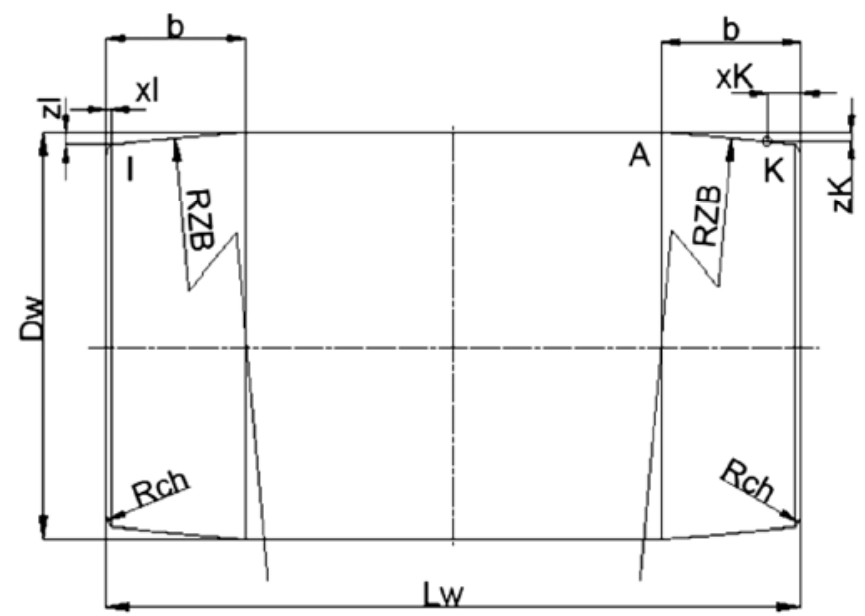

Fig. 1 Cylindrical crowned roller.

For usual applications, and rollers made of steel the profile functions $P(x)$ are presented in international standards [3] and, with some modifications, in technical documentations of bearings manufacturers.

For rollers having a length, $L_{w e} \leq 2.5 * D_{w e}$, the international standard ISO 16281 [3] gives:

$$
P(x)=0.00035 D_{w e} \ln \left[\frac{1}{1-\left(2 x / L_{w e}\right)^{2}}\right]
$$

Other commendation aims the minimum value $Z_{T_{-} \min }$ for the end relief in a given point $T$ of the minimum function $P_{\min }(x)$ :

$$
P_{\min }\left(x_{T}\right)=Z_{T-\min }
$$

Using function $P_{\min }(x)$ the profile functions $P_{\text {nom }}(x)$ and $P_{\text {max }}(x)$ are established for nominal and maximum profiles, repectively. In this paper was considered: $P_{\text {nom }}(x)=$ $1.5 * P_{\min }(x)$ and $P_{\max }(x)=2.0 * P_{\min }(x)$, Figure 2 .

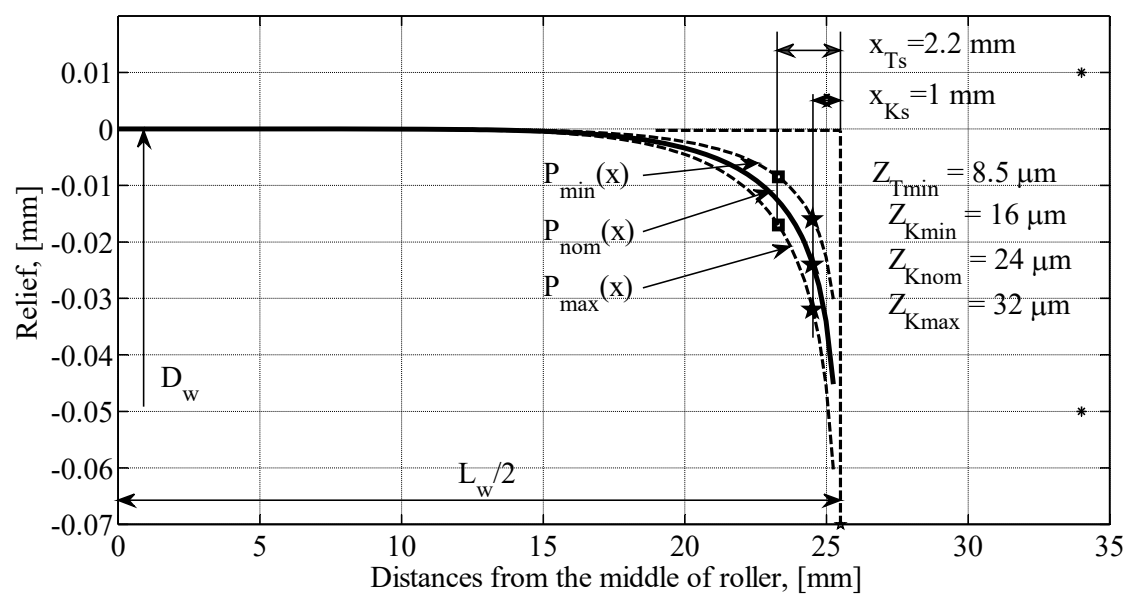

Fig. 2. The nominal profile shape $P_{\text {nom }}(x)$, function curves $P_{\min }(x), P_{\max }(x)$ and end relievs for logarithmic profiled cylindrical roller components of $\mathrm{NJ} 2320 \mathrm{E}$ bearing. 


\section{Length of circular profile}

The value of $b$ is established to maintain high values for the basic reference rating lives when the operating parameters might significantly change: from medium loads and reduced misalignments $\left(\mathrm{F}_{\mathrm{r}}=0.1 * \mathrm{C}_{\mathrm{r}, \Psi}=1 \mathrm{~min}\right.$.) till very high loads and severe misalignments $\left(\mathrm{F}_{\mathrm{r}}=\right.$ $\left.0.3{ }^{*} \mathrm{C}_{\mathrm{r}}, \Psi=3.0 \mathrm{~min}\right)$. A numerical study has been accomplished to point out the dependence of the basic reference rating lives $L_{10 \_} r$ on length $b$ of roller generatrix with circular profile.

\subsection{Rating Lives}

\subsubsection{Modified rating life}

To evaluate the modified rating life of bearings ISO 281 [8] uses the equation:

$$
L_{10 \mathrm{~m}}=a_{1} a_{\mathrm{ISO}} \cdot L_{10}=a_{1} \cdot a_{\mathrm{ISO}} \cdot\left(\frac{c_{r}}{F_{r}}\right)^{p}
$$

where $P$ is the dynamic equivalent radial load, $\mathrm{p}=10 / 3$ and $a_{1}=1$ when a $90 \%$ reliability is admitted. The life modification factor $a_{\mathrm{ISO}}$ is:

$$
a_{\mathrm{ISO}}=f\left(\frac{e_{\mathrm{C}} \cdot C_{u}}{P}, \kappa\right)
$$

where $C_{u}$ is the fatigue load limit, whereas the parameters $e_{C}$ and $\kappa$ take into account the contamination and lubrication conditions. For mineral oil lubrication and bearing raceway surfaces machined with good manufacturing quality, the condition of lubricant separation is described by the complex parameter $\kappa$, defined as the ratio of the actual kinematic viscosity $v$ to the reference kinematic viscosity $v_{1}$.

\subsubsection{Basic reference rating life. The lamina technique}

Additional to the influencing parameters described by ISO 281 [8], the standard ISO 16281 [3] takes into account three more operating parameters: the misalignment, operating internal clearance and internal load distribution on rolling elements. The main steps to obtain the basic reference rating life $L_{10 \_r}$ [3] are mentioned in the following:

Pressures distributions and distribution of contact stresses. The roller-raceway contacts of length $L_{w e}$ are divided into a number of $n_{\mathrm{s}}$ laminae each of width $w$, and $L_{w e}=n_{\mathrm{s}} \cdot w$. A number of laminae $n_{\mathrm{s}}=256$ was used in calculations. The distribution of contact stresses $q_{j, k}$ over the length of the $j$-roller is established using the pressure distribution provided by Non-Hertz semi-analytical method described in $[9,10]$. The virtual rectangular contact area was discretized with $256^{*} 64$ patches, each of them supporting a different unknown pressure. More details are in $[11,12]$. The influence of misalignment on pressures distributions achieved in the contact between the most loaded roller and inner ring of NJ2320 bearing is comparatively exemplified for operating conditions without misalignment $\psi=0$ Figure 3, and with a misalignment $\psi=1$ minute, Figure 4.

Functions for stress concentrations stresses. Approximated functions of the stress concentration stresses over the roller length are further developed for the inner ring raceway $f_{i}[j, k]$, and outer ring raceway $f_{e}[j, k][3]$.

Dynamic equivalent load on a lamina. The dynamic equivalent load on a lamina $k$ of a rotating inner ring is:

$$
q_{k e i}=\left[\frac{1}{Z_{w}} \sum_{1}^{Z_{w}}\left(f_{i}[j, k] \cdot q_{j, k}\right)^{4}\right]^{1 / 4}
$$



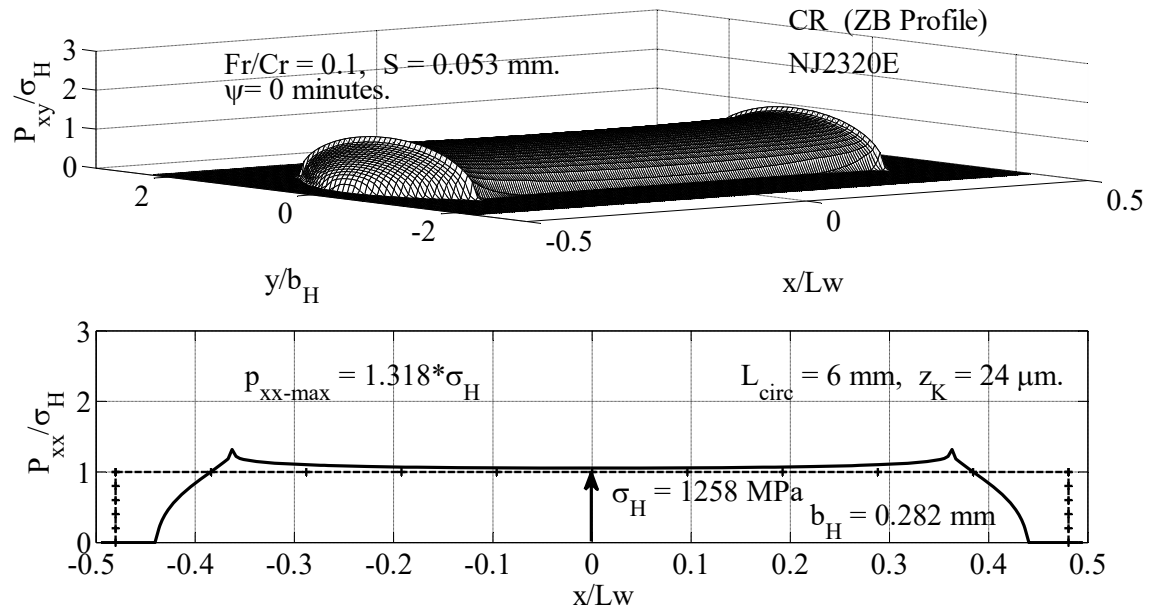

Fig. 3. Pressures distribution for operating conditions without misalignment.
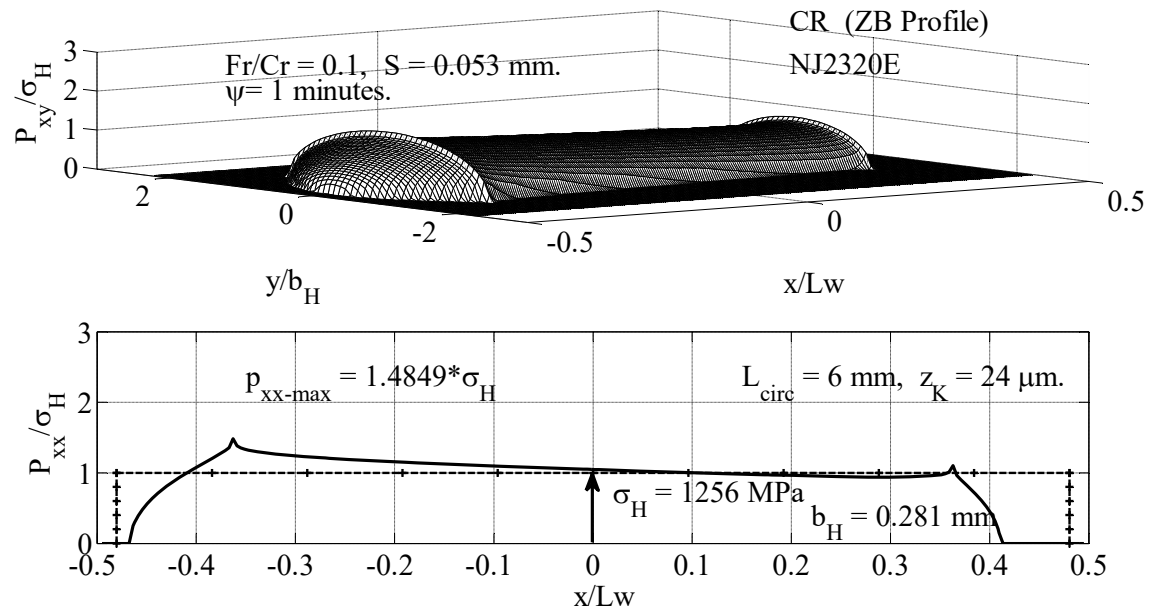

Fig. 4. Pressures distribution for operating conditions with $\psi=1$ minute misalignment.

The dynamic equivalent load on a lamina $k$ of a stationary outer ring is:

$$
q_{k e e}=\left[\frac{1}{Z_{w}} \sum_{1}^{Z_{w}}\left(f_{e}[j, k] \cdot q_{j, k}\right)^{4.5}\right]^{1 / 4.5}
$$

Fatigue lives of roller-raceway contact laminae. Considering a fourth power load-life dependence for the line contact, the fatigue lives of roller-raceway contact laminae is:

$$
L_{i j k}=\left(\frac{q_{c i j}}{q_{i j k}}\right)^{4}, L_{e j k}=\left(\frac{q_{c e j}}{q_{e j k}}\right)^{9 / 2}
$$

The basic reference rating life $L_{10 \_} r$ is calculated using the primary life formula:

$$
L_{10 \_r}=\left\{\sum_{k=1}^{m}\left[\left(\frac{q_{k c i}}{q_{k e i}}\right)^{-4.5}+\left(\frac{q_{k c e}}{q_{k e e}}\right)^{-4.5}\right]\right\}^{-8 / 9}
$$




\subsubsection{Modified reference rating life $\boldsymbol{L}_{\boldsymbol{n} \mathbf{m r}}$}

The modification factor $\boldsymbol{a}_{\text {ISo, }}$, calculated using equations (34) to (36) from [8], is determined, for each lamina and considered in equation of modified reference rating life:

$$
L_{n \mathrm{mr}}=a_{1}\left(\sum_{k=1}^{n_{s}}\left\{\left[a_{\mathrm{ISO}}\left(\frac{e_{C} \cdot C_{\mathrm{ur}}}{P_{\mathrm{ks}}}, \kappa\right)\right]^{-9 / 8} \cdot\left[\left(\frac{q_{k \mathrm{ci}}}{q_{k \mathrm{ei}}}\right)^{-4.5}+\left(\frac{q_{k \mathrm{ce}}}{q_{k \mathrm{ee}}}\right)^{-4.5}\right]\right\}\right)^{-8 / 9}
$$

The dependence of the basic reference rating life on length of circular profile

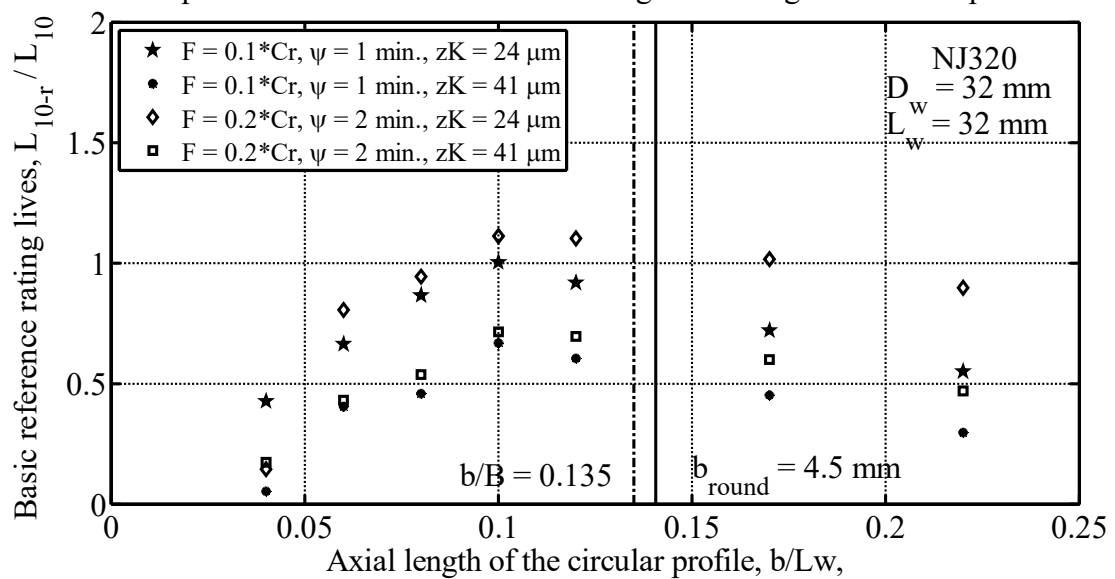

Fig. 5. The basic reference rating lives as function of length of circular profile, NJ320 bearing.

The following formulae has been derived for the minimum length of $t$ circular profiles:

$$
\begin{gathered}
b_{\text {adim }}=0.125+0.01 * \frac{L_{w}}{D_{w}} \\
b_{\text {min }}=\operatorname{round}\left(b_{\text {adim }} * L_{w}, 0.5\right)
\end{gathered}
$$

The dependence of the basic reference rating life on length of circular profile

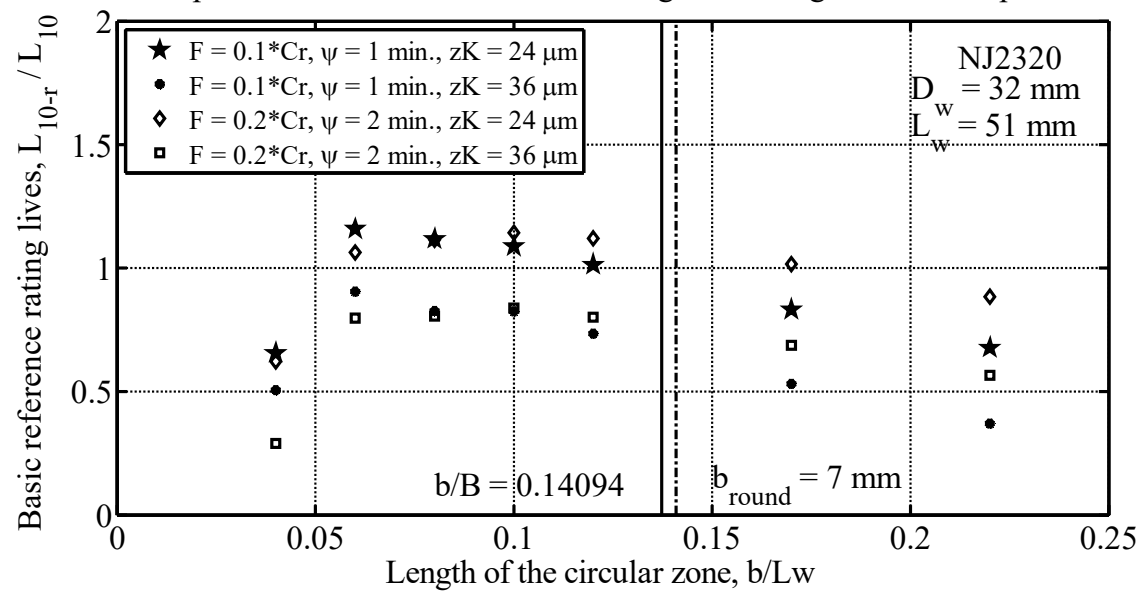

Fig. 6. The basic reference rating lives as function of length of circular profile, NJ2320 bearing.

\subsection{Basic reference rating lives and the length of circular profile}

The various operating conditions were considered as the input data in the computations of basic reference rating life $\boldsymbol{L}_{\mathbf{1 0} \_} r$. 
The values for length $b$ have been selected in order to maintain high values of basic reference rating lives for a large field of operating parameters.

Some results of computations are exemplified in Figure 5 for NJ2320 bearing and in Figure 6 for NJ320 bearing. The positions of the minimum length $b_{\text {min }}$ inside the lives dependencies plots are also presented in Figure 5 and Figure 6.

\section{Conclusions}

The size of cylindrical roller is determined by diameter $D_{w}$ and length $L_{w}$. The cylindrical crowned profile is defined by two parameters: the value $\mathrm{ZK}$ of the end relief, and the length $b$ of circular profile.

For any particular roller size $\left(D_{w} * L_{w}\right)$, the end relief $\mathrm{Z}_{\mathrm{K}}$ of cylindrical crowned profile is established considering the values provided by preferred logarithmic profile.

A numerical study has been accomplished to reveal the dependence of the basic reference rating life, $L_{10 \_r}$ on the length $b$ of roller generatrix with circular profile.

The value of $b$ was established to maintain the high values for the basic reference rating lives for a large field of operating conditions: from usual loads and misalignment $\left(\mathrm{F}_{\mathrm{r}}=0.1{ }^{*} \mathrm{C}_{\mathrm{r}}\right.$, $\Psi=1 \mathrm{~min}$.) till very high loads and severe misalignment $\left(\mathrm{F}_{\mathrm{r}}=0.3{ }^{*} \mathrm{C}_{\mathrm{r}}, \Psi=3.0 \mathrm{~min}\right)$.

\section{References}

1. E. Ioannides, G. Bergling, A. Gabelli, Acta Scandinavica, Me 137 1-80 (1999)

2. T. Harris and M. Kotzalas, Rolling bearing analysis-Advanced concepts of bearing technology (Boca Raton: CRC Press, Taylor \& Francis Group, 2007)

3. ISO 16281, Rolling bearings-methods for calculating the modified reference rating life for universally loaded bearings, Switzerland (2008)

4. S. Crețu, M. Benchea, A.Iovan-Dragomir, J. Balkan. Tribol. Assoc., 221 (2016)

5. H. Reusner, Ball Bearing Journal. 230 2-10 (1987)

6. J. de Mul, J. Kalker, B. Fredriksson, ASME J. Tribol., 108 140-148 (1986)

7. H. Fujiwara, T. Kobayashi, T. Kawase, K. Yamauchi, Tribol. Trans. 53, 910-916 (2010)

8. ISO 281, Rolling bearings-Dynamic load ratings and rating life, , Switzerland, (2007)

9. I. Polonsky, and L. Keer, ASME J. Tribol. 122, 1 (2000)

10. S. Creţu, E. Antalucă, O. Creţu, Annals Univ. Galaţi Romania 24, 26-35 (2003)

11. S. Creţu, Bull. Inst. Polit. Iași, LI (LV) 1-2, 1-31 (2005)

12. S. Creţu, Elastic plastic concentrated contact (in Romanian) (Ed. Politehnium, Iaşi, Romania, 2009) 